\title{
Wafer-Fused Optically Pumped VECSELs Emitting in the 1310-nm and 1550-nm Wavebands
}

\author{
A. Sirbu, ${ }^{1}$ N. Volet, ${ }^{1}$ A. Mereuta, ${ }^{1}$ J. Lyytikäinen, ${ }^{2}$ J. Rautiainen, ${ }^{2}$ O. Okhotnikov, ${ }^{2}$ J. Walczak, ${ }^{3}$ \\ M. Wasiak, ${ }^{3}$ T. Czyszanowski, ${ }^{3}$ A. Caliman, ${ }^{1}$ Q. Zhu, ${ }^{1}$ V. Iakovlev, ${ }^{1}$ and E. Kapon ${ }^{1}$
}

${ }^{1}$ Swiss Federal Institute of Technology (EPFL), Laboratory of Physics of Nanostructures, 1015 Lausanne, Switzerland

${ }^{2}$ Optoelectronics Research Centre, Tampere University of Technology, 33720 Tampere, Finland

${ }^{3}$ Institute of Physics, Technical University of Łódz, 90-924 Łódz, Poland

Correspondence should be addressed to A. Sirbu, alexei.sirbu@epfl.ch

Received 1 June 2011; Accepted 9 August 2011

Academic Editor: Krassimir Panajotov

Copyright ( 2011 A. Sirbu et al. This is an open access article distributed under the Creative Commons Attribution License, which permits unrestricted use, distribution, and reproduction in any medium, provided the original work is properly cited.

\begin{abstract}
1300-nm, 1550-nm, and 1480-nm wavelength, optically pumped VECSELs based on wafer-fused InAlGaAs/InP-AlGaAs/GaAs gain mirrors with intracavity diamond heat spreaders are described. These devices demonstrate very low thermal impedance of $4 \mathrm{~K} / \mathrm{W}$. Maximum CW output of devices with 5 groups of quantum wells shows CW output power of $2.7 \mathrm{~W}$ from $180 \mu \mathrm{m}$ apertures in both the $1300-\mathrm{nm}$ and the $1550-\mathrm{nm}$ bands. Devices with 3 groups of quantum wells emitting at $1480 \mathrm{~nm}$ and with the same aperture size show CW output of $4.8 \mathrm{~W}$. These VECSELs emit a high-quality beam with $M^{2}$ beam parameter below 1.6 allowing reaching a coupling efficiency as high as $70 \%$ into a single-mode fiber. Maximum value of output power of $6.6 \mathrm{~W}$ was reached for $1300 \mathrm{~nm}$ wavelength devices with $290 \mu \mathrm{m}$ aperture size. Based on these VECSELs, second harmonic emission at $650 \mathrm{~nm}$ wavelength with a record output of $3 \mathrm{~W}$ and Raman fiber lasers with $0.5 \mathrm{~W}$ emission at $1600 \mathrm{~nm}$ have been demonstrated.
\end{abstract}

\section{Introduction}

Vertical-cavity surface-emitting lasers (VCSELs) $[1,2]$ and vertical-external-cavity surface-emitting lasers (VECSELs) [3-5] emitting in the 1310-nm and 1550-nm wavelength bands are ideally based on gain medium grown in the InP material system, with InGaAsP or InAlGaAs quantum wells (QWs) that produce high optical gain in the spectral region from $1270 \mathrm{~nm}$ to $1610 \mathrm{~nm}$. Besides fibre-optic communications, this spectral range presents increasing interest in other fields of optics applications like gas sensors, free-space communications, biomedical, Raman optical amplifiers, and so forth. In recent years, the performance of 1310-nm and 1550-nm VCSELs has been considerably improved by the introduction of InAlGaAs QWs and tunnel junction injection, resulting in fundamental mode output close to $10 \mathrm{~mW}$ of wafer-fused VCSELs with AlGaAs/GaAs distributed Bragg reflectors (DBRs) [6]. Although emission in the $\mathrm{mW}$ range is sufficient for an important part of the mentioned applications, there is a number of emerging novel applications, such as in 1300-nm lasers for frequency doubled 650-nm high-power red lasers applied in laser displays [7] and 1300-1600-nm optical pumping of Raman amplifiers [8] at high output power levels in the range of several watts. This high optical power domain is accessible by the realization of optically pumped VECSELs that are capable of producing intense, high-quality beams from a large pumped area as initially demonstrated for shortwavelength VECSELs in 1999 [9]. In addition, VECSELs are suitable for insertion of optical elements inside the laser cavity not only for frequency doubling but also, for example, for subpicosecond pulse generation [10] and for reaching narrow spectral linewidths of less than $5 \mathrm{kHz}$ [11].

In order to reach the inherent advantage of VECSELs, which is the possibility of power scaling with increasing the pump area, an efficient mechanism for heat dissipation from the active region is of paramount importance. Thus, the thermal impedance of the VECSEL gain mirror that includes a semiconductor active region placed on a DBR is a quality factor that finally determines the output power level of the device. In the case of long-wavelength VECSEL gain mirrors that incorporate a DBR formed in the same 
material system as the multi-QW (MQW) active region, the heat dissipation through the InP-based quaternary alloys (InGaAsP or InAlGaAs) that form the DBR is hampered by the very low thermal conductivity of these materials, resulting in thermal impedance as high as $470 \mathrm{~K} / \mathrm{W}$ [3]. One solution to this problem consists of replacing the as-grown InP-based DBR with an AlGaAs/GaAs DBR with better thermal conductivity either in the form of a metamorphic mirror grown on the InP-based MQW active region [4] or bonded to the active region by wafer fusion as implemented in wafer-fused long-wavelength VCSELs [5]. In addition, an intracavity diamond heat-spreader that is directly bonded to the top side of the active region is very effective in considerably improving the heat dissipation in the gain mirror [12].

In this work, we present the fabrication and characterization results of wafer-fused InAlGaAs/AlGaAs optically pumped long-wavelength VECSELs with intracavity diamond heat-spreaders. We demonstrate thermal impedance as low as $4 \mathrm{~K} / \mathrm{W}$, room-temperature (RT) CW output power values of $2.7-6.6 \mathrm{~W}$ and high-quality beam emission with $70 \%$ coupling efficiency into single-mode fibres.

\section{Gain Mirror Development}

2.1. 1550-nm Wavelength Gain Mirrors. The active regions of the 1550-nm VECSELs were grown by low-pressure metal-organic vapour phase epitaxy (LP MOVPE) on (100)InP substrates as previously described in [13]. The periodic gain structures comprise 5 groups of 2 compressively strained (1\%) $\mathrm{Al}_{0.06} \mathrm{Ga}_{0.26} \mathrm{In}_{0.68} \mathrm{As} \mathrm{QWs}$. The QWs are sandwiched between tensile strained $(-0.5 \%), 10$ $\mathrm{nm}$ thick $\mathrm{Al}_{0.16} \mathrm{Ga}_{0.38} \mathrm{In}_{0.46}$ As barriers and lattice-matched $\mathrm{Al}_{0.23} \mathrm{Ga}_{0.24} \mathrm{In}_{0.53}$ As spacers and are positioned at the antinodes of the standing wave of the electromagnetic field confined by a $3 \lambda$-thick subcavity. Figure 1 shows the schematic structure of the gain medium, and the calculated near field intensity profile superimposed on the refractive index distribution of the core of the gain mirror. The photoluminescence (PL) spectrum of the active region is peaked near $1520 \mathrm{~nm}$, at room temperature (cf. Figure 2).

With increasing temperature, the PL intensity decreases, and the spectral peak shifts to longer wavelengths at a rate of $0.6 \mathrm{~nm} / \mathrm{K}$ due the temperature variation of the bandgap energy in the QWs. This material system shows quite efficient high-temperature luminescence at temperatures as high as $130^{\circ} \mathrm{C}$, which resulted in high performance, state of the art VCSELs [14]. The DBR was grown by solid-source MBE as previously described in [15] and consists of 35 pairs of quarter wave thick $\mathrm{Al}_{0.9} \mathrm{Ga}_{0.1}$ As and GaAs layers with a highreflectivity stop band centered at $1570 \mathrm{~nm}$. The fused gain mirror cavity emission spectrum has a room temperature maximum at $1570 \mathrm{~nm}$ (cf. Figure 3 ). The spectra on Figure 3 were acquired under optical pumping with a 980-nm pump laser emitting $65 \mathrm{~mW}$ under $200 \mathrm{~mA}$ driving current. The shift of the cavity emission peak with temperature is $0.23 \mathrm{~nm} / \mathrm{K}$ and is mainly due to the temperature variation of the refractive index in the active region.
2.2. 1300-nm Wavelength Gain Mirrors. The active regions of 1300-nm wavelength gain mirrors were also grown by LP MOVPE. The periodic gain structure consists of $5 \times$ 2 compressively strained (1\%) $\mathrm{A}_{0.14} \mathrm{Ga}_{0.18} \mathrm{In}_{0.68}$ As quantum wells. The QWs are sandwiched between $\mathrm{Al}_{0.28} \mathrm{Ga}_{0.26} \mathrm{In}_{0.46} \mathrm{As}$ tensile strained compensation barriers as in previously reported $1310 \mathrm{~nm}$ band VCSELs [16]. The QW pairs are separated by lattice-matched $\mathrm{Al}_{0.31} \mathrm{Ga}_{0.16} \mathrm{In}_{0.53}$ As spacers. An InP layer on the surface acts as a window layer (cf. Figure 4). The DBR, grown by MBE on a GaAs substrate, comprises 35 pairs of quarter-wave thick $\mathrm{Al}_{0.9} \mathrm{Ga}_{0.1}$ As and GaAs layers.

The PL spectrum at room temperature of this MQW active region, with a full width at half maximum of $60 \mathrm{~nm}$, is centered at $1263 \mathrm{~nm}$ (cf. Figure 5). A subcavity resonance dip of the fused gain mirror situated at $1315 \mathrm{~nm}$ within the highreflectivity stop band sets a detuning of $52 \mathrm{~nm}$ (cf. Figure 6).

2.3. 1480-nm Wavelength Gain Mirrors. Both the active MQW structure and the DBR for the 1480-nm wavelength devices were grown by a solid-source MBE [17].Compressively strained (1\%) $7 \mathrm{~nm}$ thick $\mathrm{Al}_{0.1} \mathrm{Ga}_{0.22} \mathrm{In}_{0.68}$ As QWs were sandwiched between $10 \mathrm{~nm}$ thick $\mathrm{Al}_{0.22} \mathrm{Ga}_{0.31} \mathrm{In}_{0.47} \mathrm{As}$ strain compensation barriers and separated by $\mathrm{Al}_{0.31} \mathrm{Ga}_{0.16}$ $\mathrm{In}_{0.53} \mathrm{As} / \mathrm{InP}$ spacers (cf. Figure 7 ). The topmost InP window layer of the gain section provides additional carrier confinement and prevents carrier diffusion to and subsequent nonradiative recombination at the surface. As for the designs discussed earlier, the QWs are located at the antinodes of the standing wave pattern of the electromagnetic field intensity. Distribution of the QWs was changed to 4-2-2 in order to improve pump absorption efficiency; moreover, the more compact subcavity allowed better heat extraction.

The room temperature PL spectrum of the QW structure peaked near $1445 \mathrm{~nm}$ with a full-width at half maximum of $71 \mathrm{~nm}$, as illustrated in Figure 8. The DBR is comprised of 35 pairs of quarter-wave thick $\mathrm{Al}_{0.9} \mathrm{Ga}_{0.1} \mathrm{As} / \mathrm{GaAs}$ layers with the high-reflectivity stop band centered at $1490 \mathrm{~nm}$. The dip at the center of the stop band is caused by the absorption of the QWs.

2.4. Gain Mirror Assembled by Wafer Fusion. The InAlGaAs/ InP active regions grown on InP substrates and the AlGaAs/ GaAs DBRs grown on the GaAs substrates are fused together to form long-wavelength gain mirrors using the wafer fusion technique. In the standard fusion process that was previously developed for the fabrication of long-wavelength VCSELs [2], InP-based and GaAs-based 2-inch wafers are brought into contact at $600^{\circ} \mathrm{C}$ in vacuum, or purified nitrogen, while applying a force of $7000 \mathrm{~N}$ for $30 \mathrm{~min}$ in an industrial custom-built wafer bonding machine. At these values of temperature and pressure, both wafers undergo a slight plastic deformation resulting in a uniform contacting on a nanometer scale. As a result, covalent bonds are formed between the InP-based and GaAs-based wafers. After cooling down, the stack that includes the InAlGaAs/InP$\mathrm{AlGaAs} / \mathrm{GaAs}$ half cavity with $\mathrm{InP}$ and GaAs substrates on respective sides is bowed. This bowing, with a radius of curvature of about $1 \mathrm{~m}$, occurs because of different values 


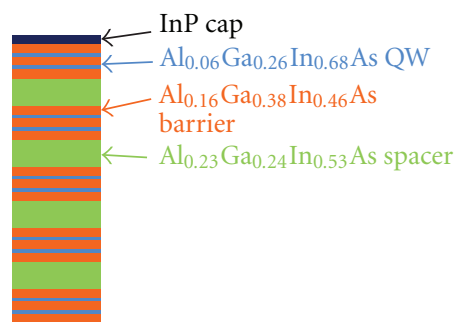

(a)

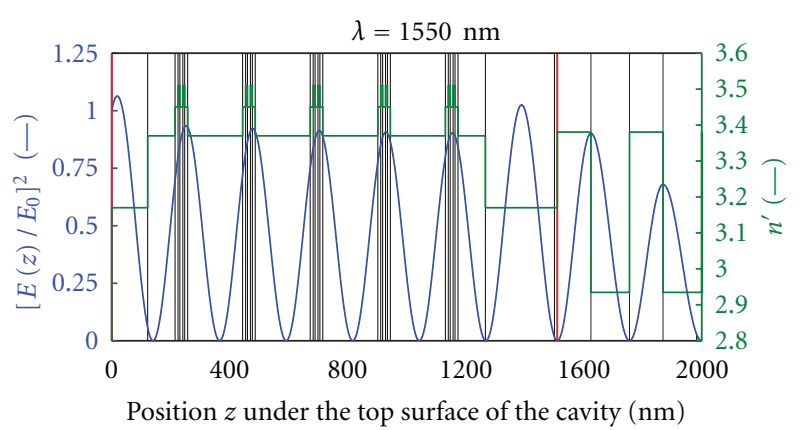

(b)

Figure 1: Schematic of layer design in the active region (a) and normalized electric field (blue line) and refractive index (green line) distributions in the 1550-nm wavelength gain mirror. Black lines show the different layers in the VECSEL subcavity (delimited by red lines) and the first 4 layers in the DBR section.

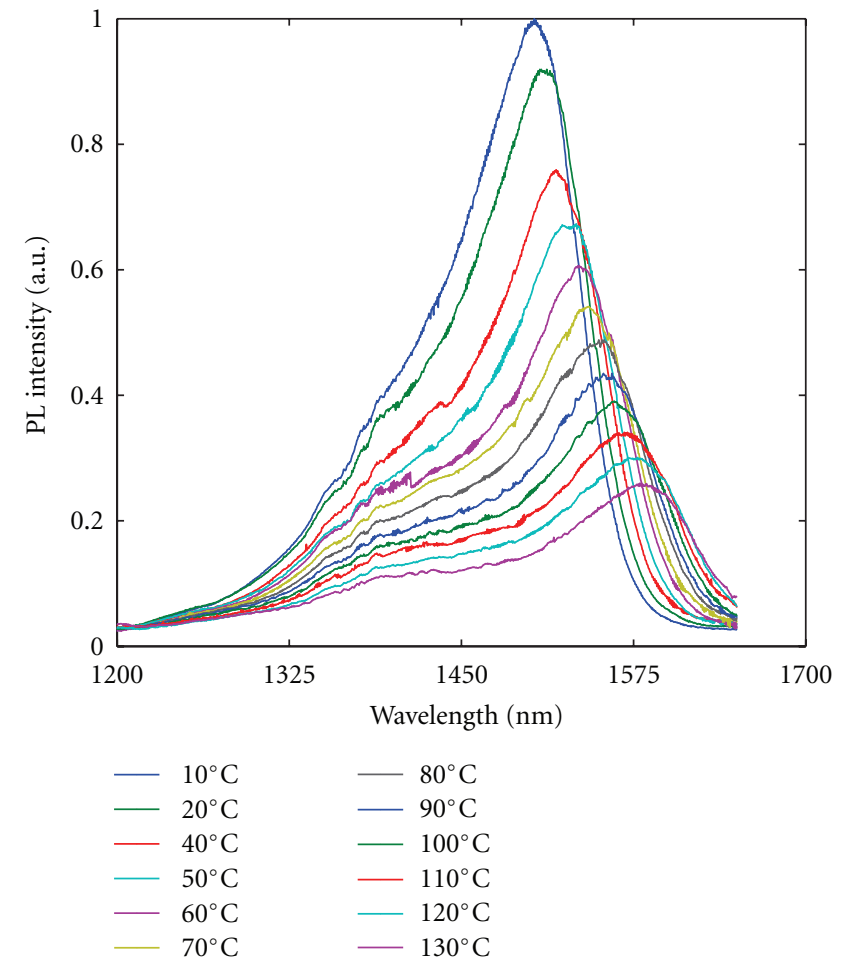

FIGURE 2: Photoluminescence spectra of the gain medium of 1550$\mathrm{nm}$ wavelength VECSELs versus temperature.

of thermal expansion coefficients of the GaAs-based wafers with lattice parameter of $5.6535 \AA$ and the InP-based wafers with lattice parameter of $5.86875 \AA,\left(5.8 \cdot 10^{-6} \mathrm{~K}^{-1}\right.$ for GaAs and $4.8 \cdot 10^{-6} \mathrm{~K}^{-1}$ for InP). After selectively etching the InP substrate, the remaining GaAs substrate containing the fused stack regains its planarity. As a result of this process, some dark-line defects are formed in the active region, which normally do not affect devices of small aperture size of about $7 \mu \mathrm{m}$. In the case of high-power VECSELs with an aperture size of the order of $100 \mu \mathrm{m}$, special care needs to be taken for decreasing the defect formation appearing due to the different thermal expansion coefficients of the GaAs-based

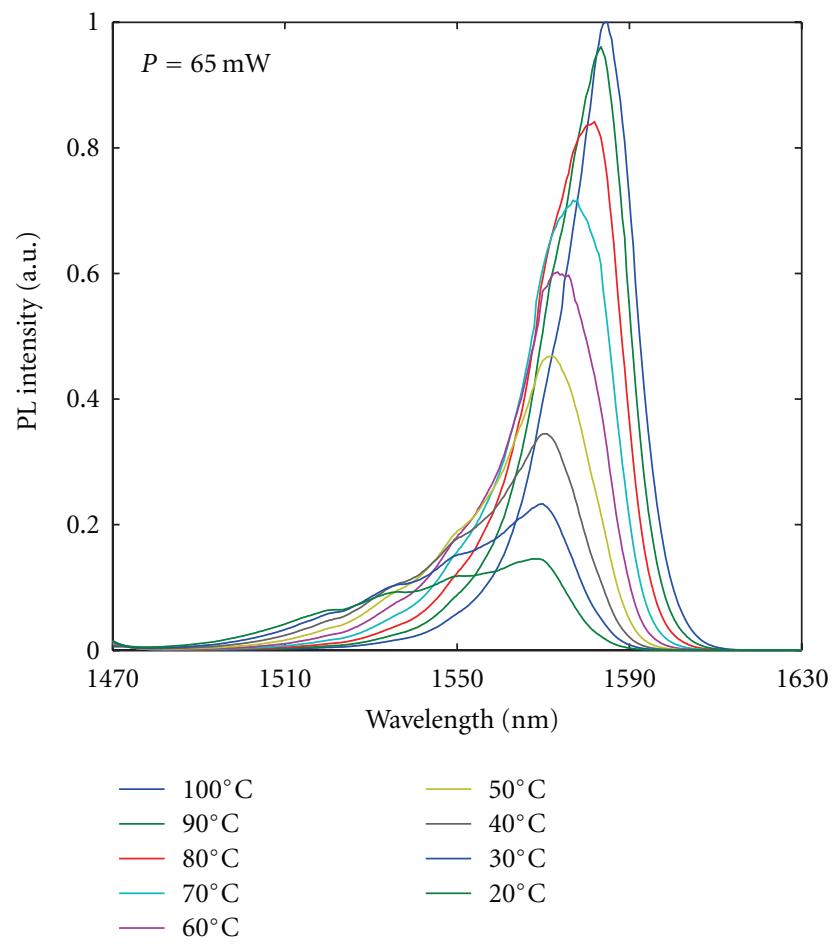

Figure 3: Gain mirror emission spectra of the 1550-nm devices versus temperature.

and InP-based wafers during cooling down from $600^{\circ} \mathrm{C}$ to RT. In this work, we applied a modified fusion technique that allows reducing the formation of dark-line defects in the active region [18]. The new approach consists of transferring the AlGaAs/GaAs DBRs on InP substrates before fusing them on the InAlGaAs/InP wafers. Thus, in the fused stack, both wafers have the same InP substrates and practically no strain is introduced in the active region during the cooling down to room temperature.

We studied a $1300 \mathrm{~nm}$ gain mirror with cathodoluminescence (CL) spectroscopy, which allows revealing defects in a field of $50 \times 50 \mu \mathrm{m}^{2}$. For CL mapping of larger areas, we 


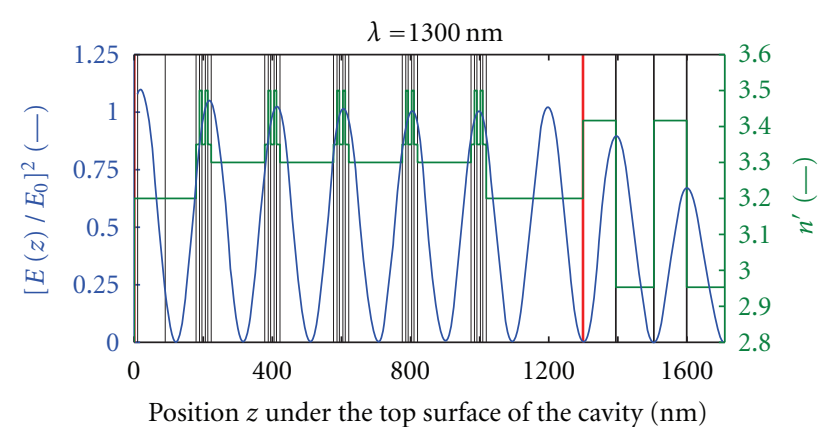

Figure 4: Normalized electric field (blue line) and refractive index (green line) distributions in the 1300-nm wavelength gain mirror. Black lines show the different layers in the VECSEL subcavity (delimited by red lines) and the first 4 layers in the DBR section.

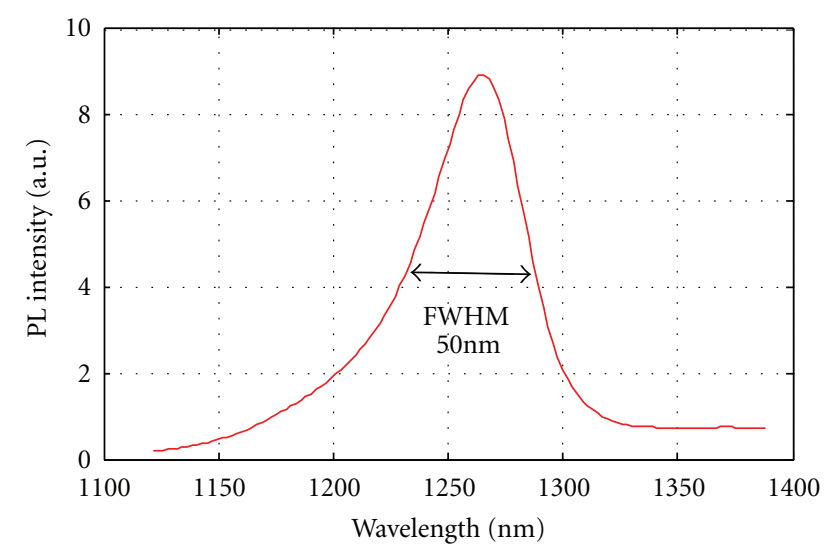

FigURe 5: PL spectrum of the 1300-nm wavelength active region at room temperature.

deposited on a $1300 \mathrm{~nm}$ InAlGaAs/InP-AlGaAs/GaAs fused gain mirror (described in Section 2.2) a grid of Ti-Au stripes with exposed cells of $25 \times 25 \mu \mathrm{m}^{2}$ and performed CL analysis of different regions of $50 \times 50 \mu \mathrm{m}^{2}$. By assembling, these images, a CL mapping of the whole sample presented on Figure 9 was reconstructed (cf. Figure 10).

In Figure 10, we can observe just one dark line on the gain mirror of $150 \times 250 \mu \mathrm{m}^{2}$ area. We can also identify 5 dark points that represent dust particles. In one cell on the left side of the sample, marked with a yellow arrow on Figure 9, the gold film did not lift off, and henc, this area appears dark in the CL image.

2.5. Heat Dissipation Scheme. Recently, two efficient heat dissipation schemes have emerged to produce high-power optically pumped VECSELs in the wavelength range of $1-3 \mu \mathrm{m}$ [19-21]. In the first approach [19], the VECSEL structure that demonstrated $20 \mathrm{~W}$ at $-20^{\circ} \mathrm{C}$ at $950 \mathrm{~nm}$ was first grown in a reverse order on a GaAs substrate, then, after soldering it to a diamond heat spreader, the GaAs substrate was selectively etched. In the second approach $[20,21]$ that has been adopted in the current work, effective heat dissipation is obtained by introducing an optically transparent intracavity diamond that is directly bonded to the gain

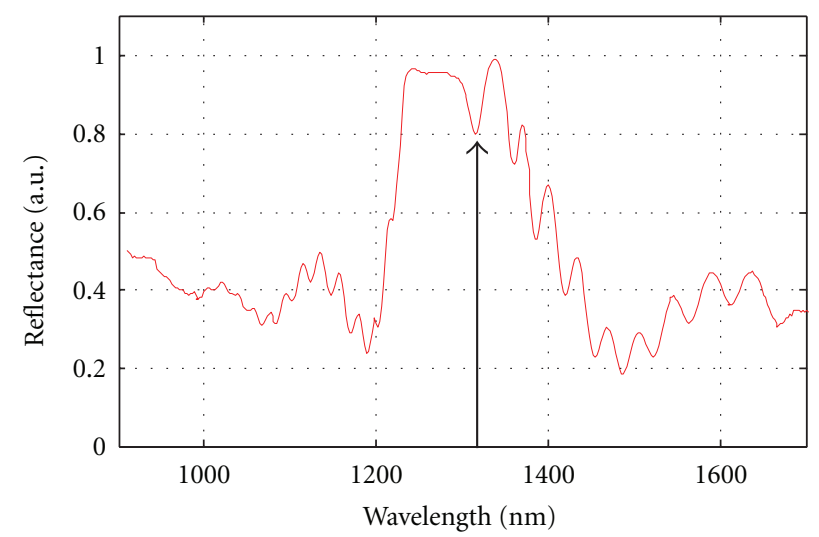

FIGURE 6: Reflectance spectrum of the 1300-nm wavelength gain mirror. Subcavity resonance at $1315 \mathrm{~nm}$ is indicated by an arrow.

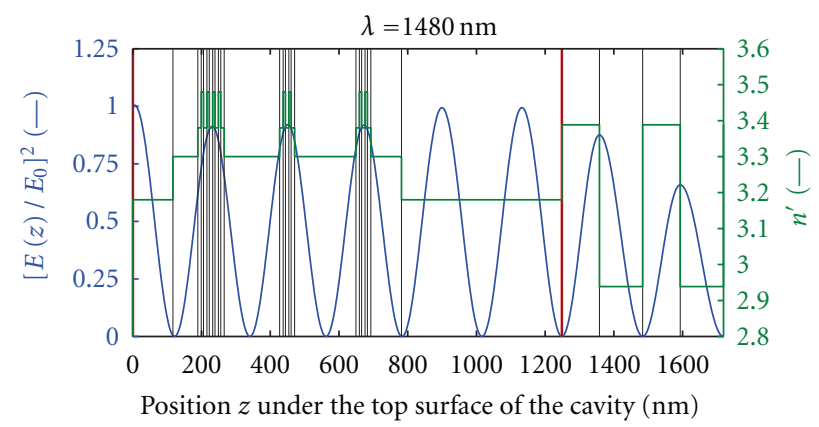

FIGURE 7: Refractive index profile and distribution of the electric field in the 1480 -nm gain mirror. Black lines show the different layers in the VECSEL subcavity (delimited by red lines) and the first 4 layers in the DBR section.

mirror. In this study, we used a $3 \times 3 \times 0.3 \mathrm{~mm}^{3}$ natural type IIa diamond that was bonded to the InP window layer of the gain mirror by molecular bonding as described in [22].

We performed thermal modelling of the wafer-fused gain mirror with diamond heat spreader mounted on a watercooled copper block by solving the thermal equation using a finite element method for a generated heat power of $3.3 \mathrm{~W}$ in the active region [18]. To simplify the calculations, the gain mirror design was transformed from 3D Cartesian to 2D cylindrical coordinate system by keeping the volumes of the active region layers unchanged. We assumed Gaussian distribution of the pumped beam in the lateral direction and exponential decay depending on the absorption coefficient of particular layers in the vertical direction. The heat generation distribution reflects the distribution of the energy absorbed by the device. Results depicted on Figure 11 demonstrate that the diamond thickness of $300 \mu \mathrm{m}$ used in this work is quite sufficient for efficient heat removal from the active region.

The distribution of the temperature within the gain mirror, depicted in Figure 12, shows that the temperature decreases quite fast with increasing distance from the pumped part of the active region in the lateral direction. The heat dissipation mechanism consists in effective heat flow from the active region into the diamond slab followed by heat 


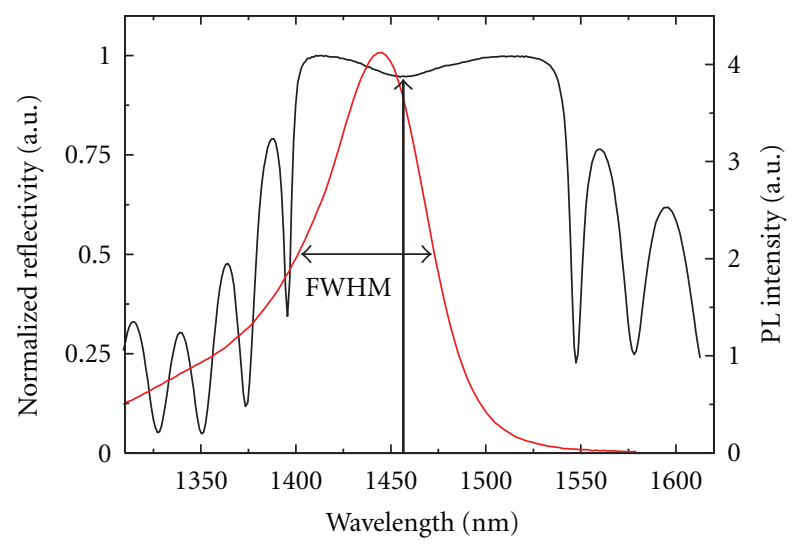

FIGURE 8: Spectral distributions of room-temperature PL of the QWs and DBR reflectivity for the 1480-nm wavelength structures.

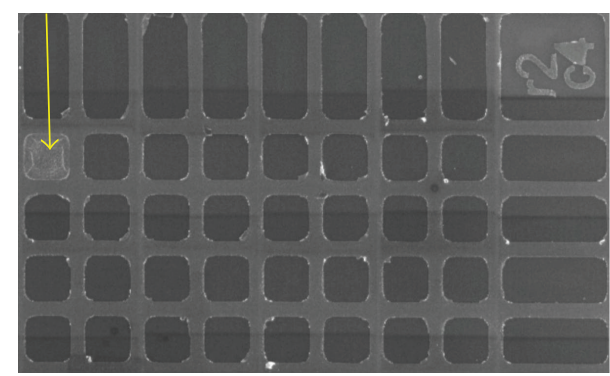

FIgURE 9: Ti/Au grid on the surface of a $1300 \mathrm{~nm}$ gain mirror sample. Yellow arrow points to a cell in which the Ti/Au did not lift off.

reinjection into the unpumped part of the active region and further dissipation through the AlGaAs/GaAs DBR.

The procedure of thermal impedance determination consists of calculating the temperature distribution within the gain mirror for three different lasing conditions: $3 \mathrm{~W}$ and $300 \mathrm{~K}, 3 \mathrm{~W}$ and $300.1 \mathrm{~K}$, and $3.01 \mathrm{~W}$ and $300 \mathrm{~K}$. The temperature distribution is further transformed into a distribution of the refractive index, and the emission wavelength is derived. The value of thermal impedance determined in such a way should be close to the top limit value estimated from direct temperature determination, since the temperature in the active region is the highest, and there is no modification of the refractive index in the region between the gain mirror and the external mirror. If one replaces the air by diamond and assumes a thermo-optic coefficient of diamond $d n / d T=0$, thermal impedance will be equal to the value determined with air. Since the thermo-optic properties of the diamond are not well known, we performed the determination of thermal impedance in a broad range of thermo-optic coefficient values of $0-2 \cdot 10^{-5} \mathrm{~K}^{-1}$. Figure 13 depicts the calculated dependence of the thermal impedance $Z$ as a function of the thermo-optic coefficient $d n / d T$. The experimental value $Z=4 \mathrm{~K} / \mathrm{W}$ (see Section 3.2) is shown as a line in Figure 13.

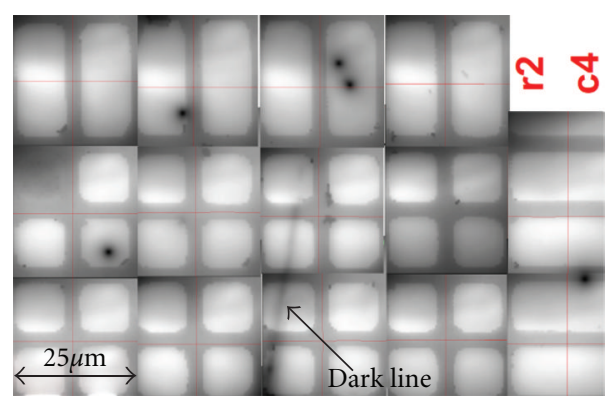

FIGURE 10: CL mapping of the sample depicted on Figure 9.

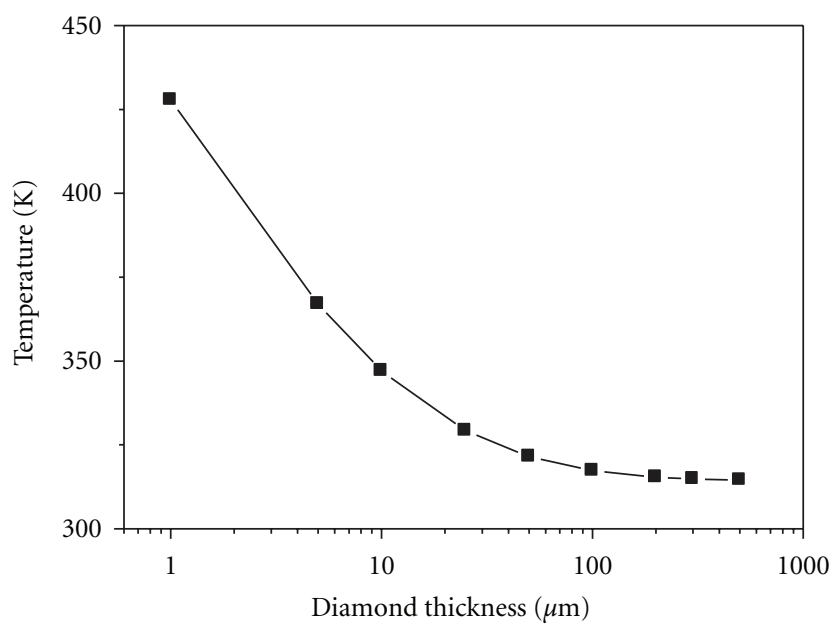

Figure 11: Maximum temperature in the active region versus diamond thickness.

\section{VECSEL Characteristics}

3.1. Optically Pumped VECSEL Cavity. Gain mirrors that are bonded to intra cavity diamond are further mounted between two copper plates with indium foil in between to ensure reliable thermal contact between the surfaces. This assembly is placed on a thermoelectric cooler mounted on a copper water-cooled block. The topmost copper plate has a circular aperture for signal and pump beams (cf. Figure 14). The lasing measurements are done with reflective plane output couplers with different values of output coupling of $1 \%-2.5 \%$. The pump source is a fibre-coupled 980 -nm diode laser. The cavity of the VECSEL is of V-type and is composed of a planar output coupler, a curved mirror and the gain mirror, as shown in Figure 15. The cavity is simulated numerically to ensure that the mode size at the gain mirror matches the pump spot.

3.2. 1550-nm VECSELs Test Results. Output characteristics of the 1550-nm wavelength VECSEL [23] for different operation temperatures are shown in Figure 18. In the temperature range of $10^{\circ} \mathrm{C}-35^{\circ} \mathrm{C}$, the output power increases up to $2.62 \mathrm{~W}$ at $10^{\circ} \mathrm{C}$ and $1.6 \mathrm{~W}$ at $35^{\circ} \mathrm{C}$. These values are limited by the maximum available input pump power of $25 \mathrm{~W}$. With increasing pump power, the emission wavelength shifts to 


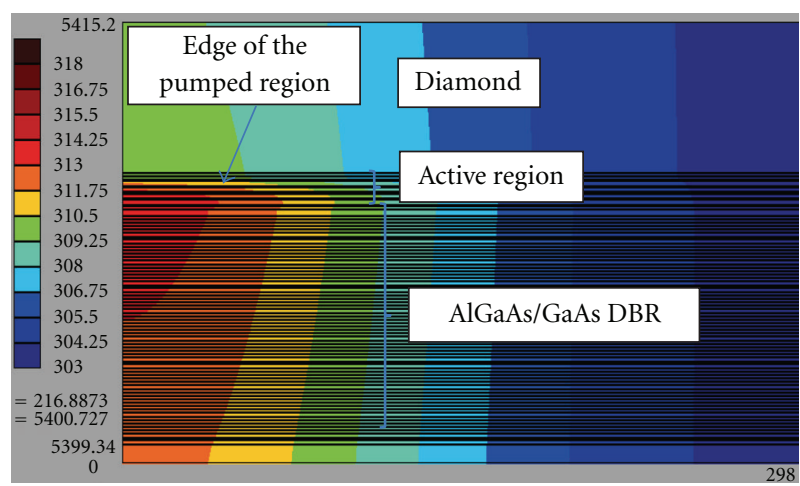

FIGURE 12: Temperature distribution in the active region.

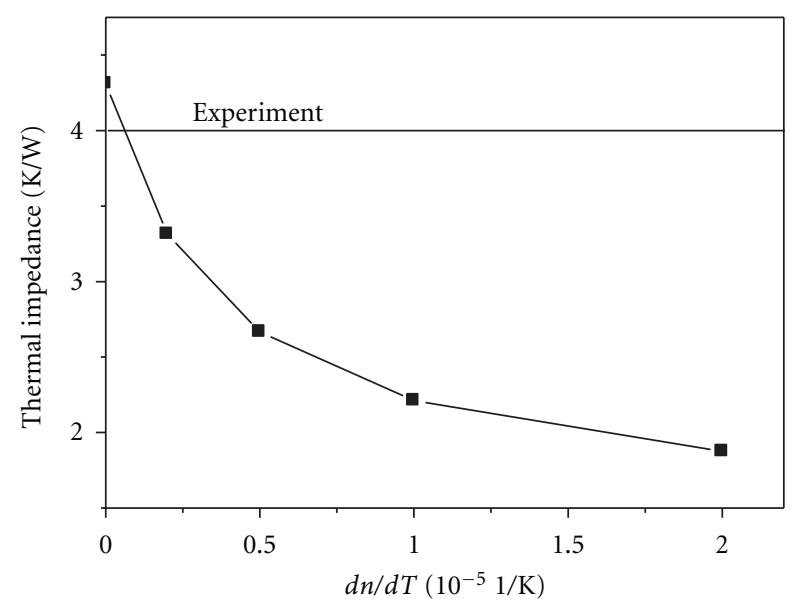

Figure 13: Thermal impedance $Z$ as a function of thermo-optic coefficient $d n / d T$.

longer wavelengths at a rate of $1 \mathrm{~nm} \cdot \mathrm{W}^{-1}$. The effective thermal impedance is estimated as $4 \mathrm{~K} \cdot \mathrm{W}^{-1}$ from the ratio of wavelength shift at different pump power levels and the wavelength shift with temperature of the gain mirror subcavity at a low pump power level determined in Section 2.1. Light-light characteristics are linear up to $15 \mathrm{~W}$ pumping power with a slope efficiency of $12 \%$ at $10^{\circ} \mathrm{C}$ (Figure 17). The recalculated efficiency inside the structure is equal to $16 \%$ when taking into consideration that the gain mirror, not containing an antireflective coating, reflects $30 \%$ of the incident pumping power. At higher pumping levels the lightlight characteristics are sublinear, which is probably due to excessive heating as a consequence of a large defect energy $\Delta E_{d}$ at $980 \mathrm{~nm}$ pumping wavelength $\left(\Delta E_{d}=0.48 \mathrm{eV}, 38 \%\right.$ of pump photons energy is heating the lattice).

3.3. 1310-nm VECSELs Test Results. Output power versus pump power characteristics of the 1310-nm VECSELs with a pump spot of $180 \mu \mathrm{m}$ diameter [24] (cf. Figure 18) demonstrate values of $1.9 \mathrm{~W}, 2.4 \mathrm{~W}$, and $2.7 \mathrm{~W}$ for output coupling of $1 \%, 2 \%$ and $2.5 \%$, respectively. Any roll-over in the output characteristics was not observed with this gain medium for pump power up to $25 \mathrm{~W}$, which limited also the achievable output power. The slope efficiency for these

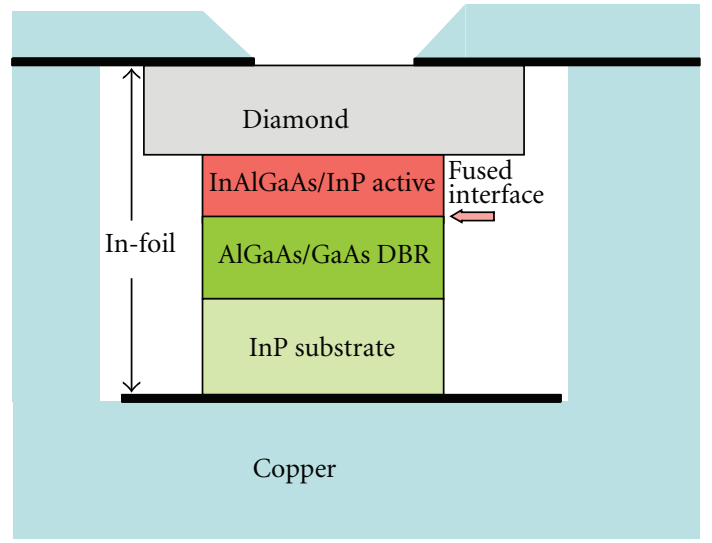

FIGURE 14: The gain mirror assembly.

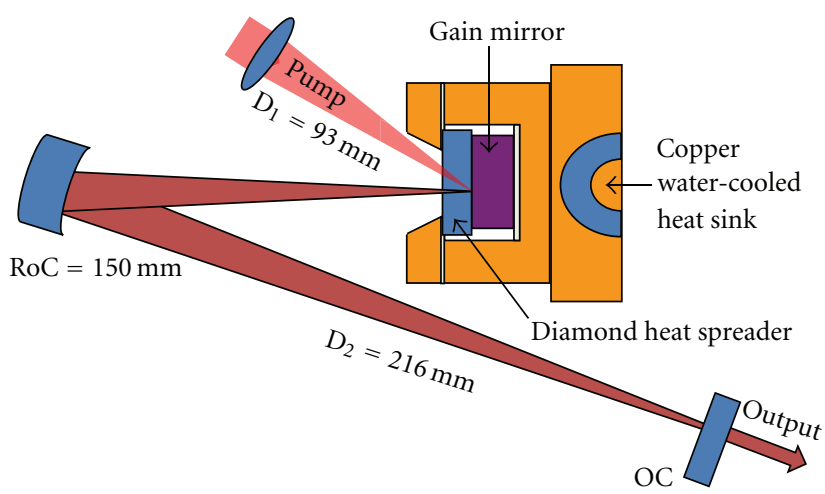

FIGURE 15: Schematic representation of the VECSEL cavity.

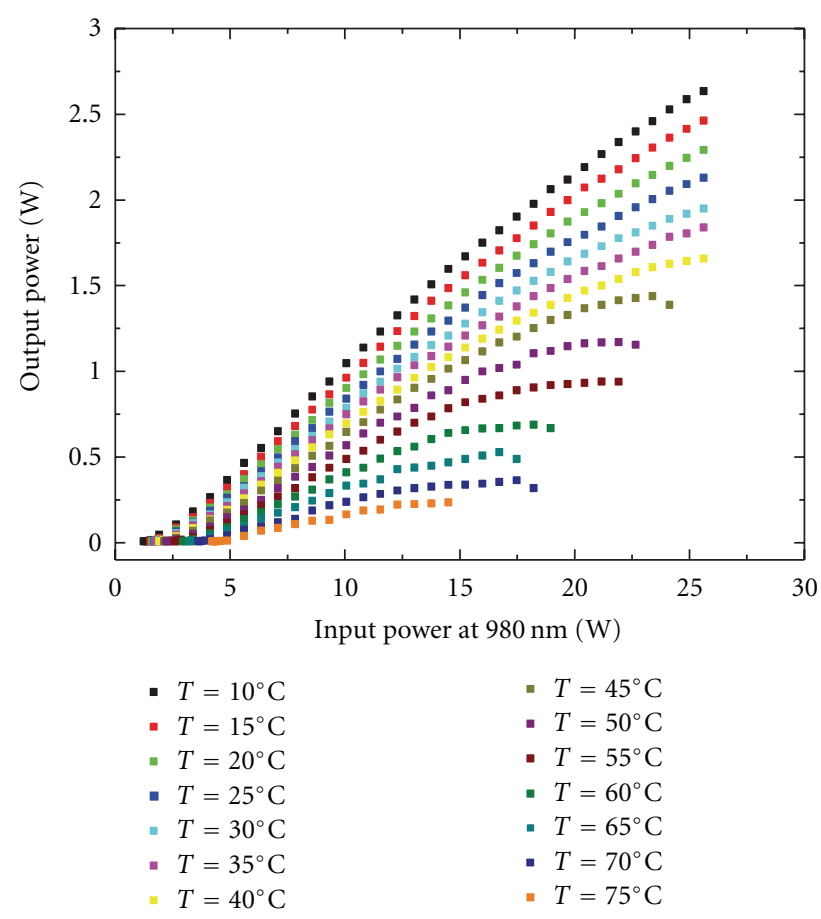

Figure 16: Output characteristics for different operating temperatures for a $1550-n m$ VECSEL. The output coupling is $2 \%$. 


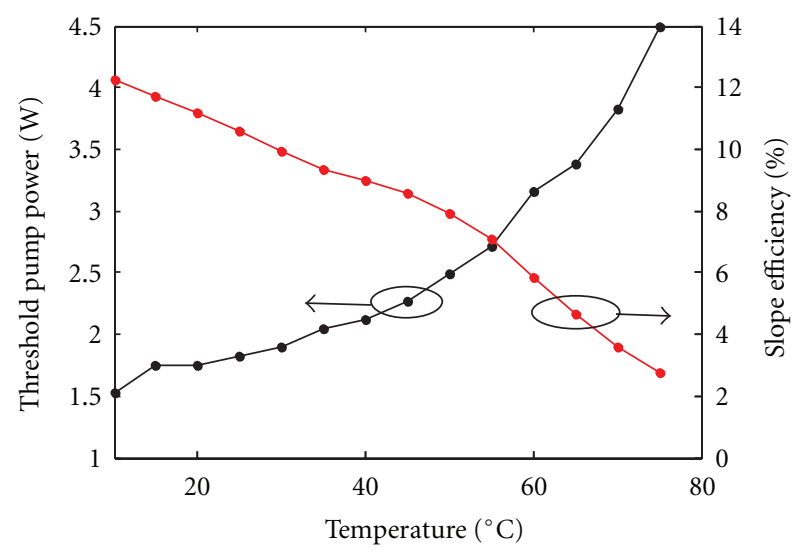

FIgUre 17: Threshold pump power and slope efficiency as a function of gain mirror temperature for the 1550-nm VECSEL.

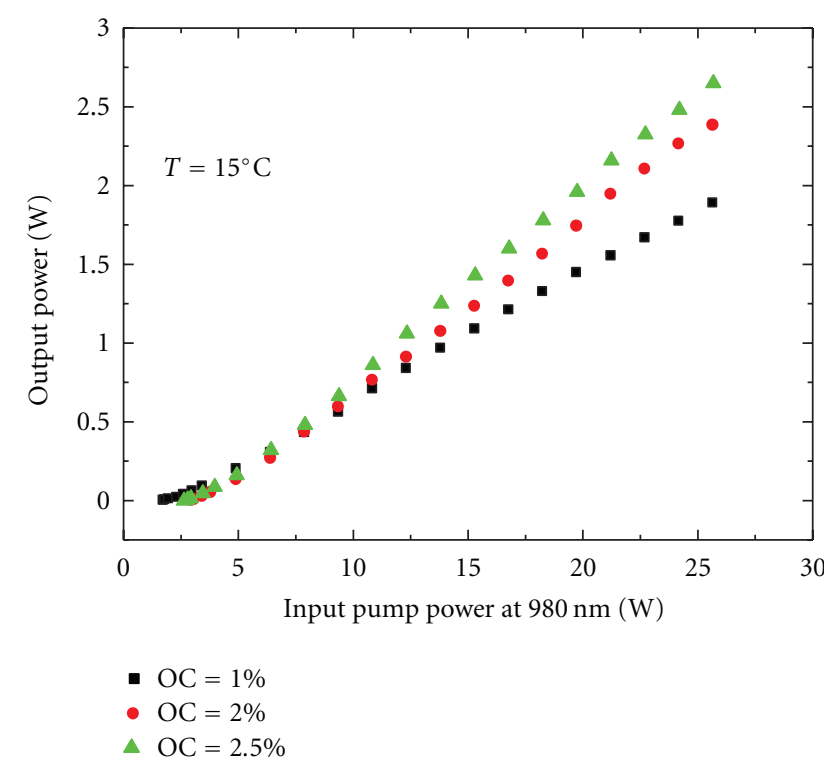

Figure 18: Output characteristics of $1310 \mathrm{~nm}$ wavelength $180 \mu \mathrm{m}$ aperture VECSEL device.

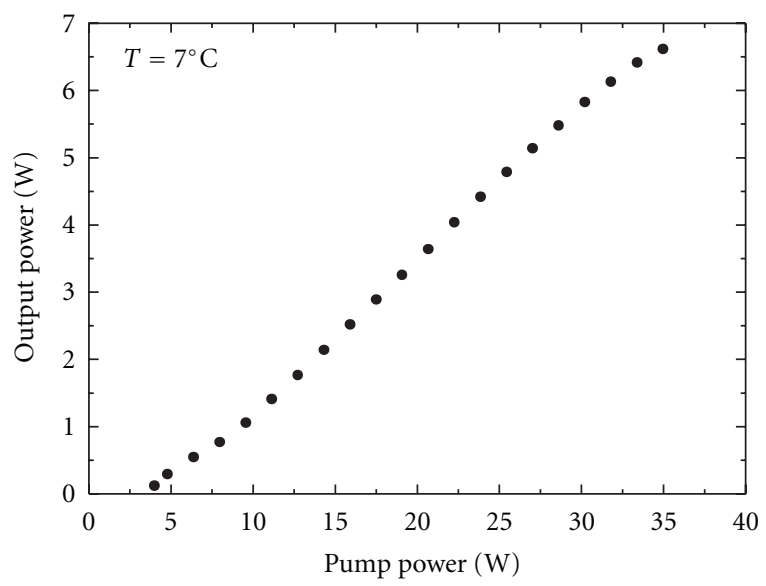

FIGURE 19: Output characteristics of the $1310 \mathrm{~nm}$ wavelength $290 \mu \mathrm{m}$ aperture VECSEL device.

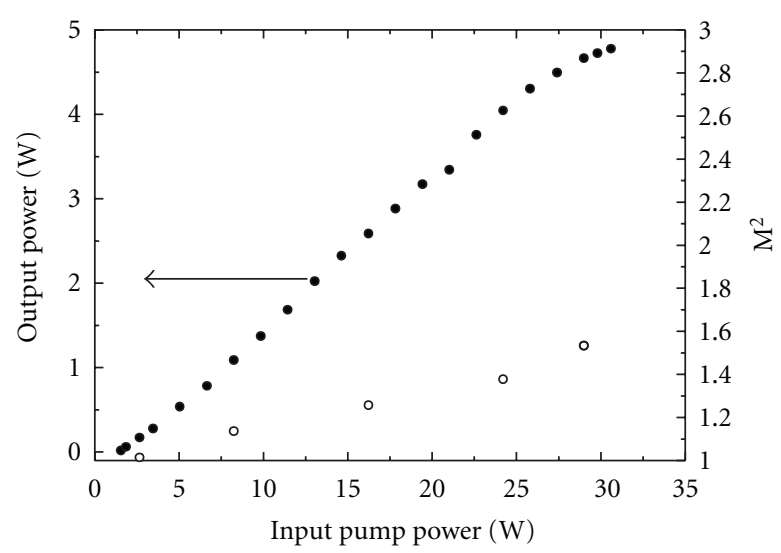

FIgURE 20: Output power and $M^{2}$ versus pump power.

devices ranges from $8.2 \%$ to $12.2 \%$ and the threshold pump power varies between $2.0 \mathrm{~W}$ and $3.1 \mathrm{~W}$.

In another test, the optical pumping beam of a $980 \mathrm{~nm}$ pump laser diode laser was focused onto a spot with a diameter of about $290 \mu \mathrm{m}$ at the gain mirror [25]. At $7^{\circ} \mathrm{C}$, the maximum output power of $6.6 \mathrm{~W}$ is reached with a $2.5 \%$ output coupler (Figure 19), which represents the highest reported value of power at this wavelength, to our knowledge. Based on these devices, second harmonic emission at $650 \mathrm{~nm}$ with a continuous wave output of $3 \mathrm{~W}$ was demonstrated for the first time [25].

3.4. 1480-nm VECSEL Results. The 1480-nm VECSELs have been tested at a pump spot diameter of $180 \mu \mathrm{m}$ to enable single transverse mode operation. Figure 20 presents the output power and $M^{2}$ beam parameter versus pump power characteristics measured with an output coupler of $2.5 \%$ at $8^{\circ} \mathrm{C}$. Maximum output power of $4.8 \mathrm{~W}$, slope efficiency of 0.18 and $M^{2}$ beam parameter below 1.6 for all pump power levels have been achieved. This high-quality emission beam allowed coupling into a single mode fiber with a coupling efficiency of $70 \%$ and a Raman fiber laser emitting $0.5 \mathrm{~W}$ at $1600 \mathrm{~nm}$ was demonstrated [26].

\section{Conclusions}

We have developed wafer-fused InAlGaAs/AlGaAs optically pumped VECSELs with intracavity diamond heat spreaders emitting at wavelengths of 1300, 1550, and $1480 \mathrm{~nm}$, based on a modified wafer-fusion process allowing reduced defect formation in the active region. These devices produce record high levels of continuous wave power of 2.7, 2.62 and $4.8 \mathrm{~W}$, respectively, with aperture diameter of $180 \mu \mathrm{m}$, and $6.6 \mathrm{~W}$ at $1300 \mathrm{~nm}$ with increased aperture diameter of $290 \mu \mathrm{m}$. A substantial increase of output power is observed when the configuration of the QWs is changed from 5 groups of 2 QWs to 3 groups of 4, 2, 2 QWs. Reaching high levels of output power as well as power scaling with increasing aperture size is possible as a result of the very low thermal impedance value of $4 \mathrm{~K} / \mathrm{W}$, which is predicted by thermal modeling and confirmed by experiment. These devices emit a high-quality 
beam with $M^{2}$ beam parameter below 1.6, allowing reaching a coupling efficiency into a single mode fiber as high as $70 \%$. Based on VECSELs developed in this work, second harmonic emission at $650 \mathrm{~nm}$ with a record output of $3 \mathrm{~W}$ and Raman fiber lasers with $0.5 \mathrm{~W}$ emission at $1600 \mathrm{~nm}$ have been demonstrated.

\section{Acknowledgment}

This work was partially supported by the Swiss Nano-Tera project MIXSEL.

\section{References}

[1] W. H. Hofmann, M. Müller, G. Böhm, M. Ortsiefer, and M.-C. Amann, " $1.55 \mu \mathrm{m}$ InP-based VCSEL with enhanced modulation bandwidths $>10 \mathrm{GHz}$ up to $85^{\circ} \mathrm{C}$," in Optical Fiber Communication (OFC) Conference, March 2009.

[2] A. Syrbu, A. Mircea, A. Mereuta et al., "1.5-mW single-mode operation of wafer-fused 1550-nm VCSELs," IEEE Photonics Technology Letters, vol. 16, no. 5, pp. 1230-1232, 2004.

[3] C. Symonds, I. Sagnes, J. L. Oudar et al., "Room temperature CW lasing operation of monolithically grown $1.55 \mu \mathrm{m}$ vertical external cavity surface emitting laser," Optics Communications, vol. 230, no. 4-6, pp. 419-423, 2004.

[4] J. P. Tourrenc, S. Bouchoule, A. Khadour et al., "Thermal optimization of $1.55 \mu \mathrm{m}$ OP-VECSEL with hybrid metalmetamorphic mirror for single-mode high power operation," Optical and Quantum Electronics, vol. 40, no. 2-4, pp. 155165, 2008.

[5] J. Lyytikäinen, J. Rautiainen, A. Sirbu et al., "Wafer fused InPGaAs optically-pumped semiconductor disk laser operating at 1.57- $\mu \mathrm{m}$," in Photon Counting Applications, Quantum Optics, and Quantum Information Transfer and Processing II, Proceedings of SPIE, April 2009.

[6] A. Caliman, V. Iakovlev, A. Mereuta, A. Sirbu, G. Suruceanu, and E. Kapon, " $8 \mathrm{~mW}$ fundamental mode output of waferfused VCSELs emitting in the 1550-nm band," in the Conference on Lasers and Electro-Optics/International Quantum Electronics (CLEO/IQEC) Conference, 2009.

[7] J. L. A. Chilla, H. Zhou, E. Weiss et al., "Blue \& green opticallypumped semiconductor lasers for display," in Projection Displays XI, Proceedings of SPIE, pp. 41-47, January 2005.

[8] M. N. Islam, "Raman amplifiers for telecommunications," IEEE Journal on Selected Topics in Quantum Electronics, vol. 8, no. 3, pp. 548-559, 2002.

[9] M. Kuznetsov, F. Hakimi, R. Sprague, and A. Mooradian, "Design and characteristics of high-power ( $>0.5-\mathrm{W}$ CW) diode-pumped vertical-external-cavity surface-emitting semiconductor lasers with circular TEM00 beams," IEEE Journal on Selected Topics in Quantum Electronics, vol. 5, no. 3, pp. 561$573,1999$.

[10] S. Hoogland, S. Dhanjal, A. C. Tropper et al., "Passively modelocked diode-pumped surface-emitting semiconductor laser," IEEE Photonics Technology Letters, vol. 12, no. 9, pp. 11351137, 2000.

[11] R. H. Abram, K. S. Gardner, E. Riis, and A. I. Ferguson, "Narrow linewidth operation of a tunable optically pumped semiconductor laser," Optics Express, vol. 12, no. 22, pp. 54345439, 2004.
[12] H. Lindberg, A. Larsson, and M. Strassner, "Single-frequency operation of a high-power, long-wavelength semiconductor disk laser," Optics Letters, vol. 30, no. 17, pp. 2260-2262, 2005.

[13] A. Mereuta, V. Iakovlev, A. Caliman et al., "High single mode power wafer fused InAlGaAs/InP - AlGaAs/GaAs VCSELs emitting in the 1.3-1.6 $\mathrm{m}$ wavelength range," in the 19th IEEE International Conference on Indium Phosphide and Related Materials (IPRM '07), pp. 430-433, May 2007.

[14] E. Kapon, A. Sirbu, V. Iakovlev, A. Mereuta, A. Caliman, and G. Suruceanu, "Recent developments in long wavelength VCSELs based on localized wafer fusion," in the 11th International Conference on Transparent Optical Networks (ICTON '09), July 2009.

[15] J. Lyytikäinen, J. Rautiainen, S. Suomalainen et al., "MBE grown optically pumped semiconductor disk lasers emitting at $940 \mathrm{~nm}$," Materials Science and Engineering B, vol. 147, no. 2-3, pp. 161-165, 2008.

[16] A. Mereuta, G. Suruceanu, A. Caliman, V. Iacovlev, A. Sirbu, and E. Kapon, "10-Gb/s and 10-km error-free transmission up to $100^{\circ} \mathrm{C}$ with $1.3-\mu \mathrm{m}$ wavelength wafer-fused VCSELs," Optics Express, vol. 17, no. 15, pp. 12981-12986, 2009.

[17] J. Lyytikäinen, J. Rautiainen, A. Sirbu et al., "High-power 1.48$\mu \mathrm{m}$ wafer-fused optically pumped semiconductor disk laser," IEEE Photonics Technology Letters, vol. 23, no. 13, pp. 917-919, 2011.

[18] A. Sirbu, A. Mereuta, A. Caliman et al., "High-power opticallypumped VECSELs emitting in the 1310-nm and 1550-nm wavebands," in Vertical External Cavity Surface Emitting Lasers, VECSELs, vol. 7919 of Proceedings of SPIE, January 2011.

[19] B. Rudin, A. Rutz, D. J. Maas et al., "Efficient Highpower VECSEL generates $20 \mathrm{~W}$ continuous-wave radiation in a fundamental transverse mode," in Advanced Solid-State Photonics (ASSP), 2009.

[20] M. Rattunde, B. Rösener, S. Kaspar et al., "GaSb-based semiconductor disk lasers for the $2-3 \mu \mathrm{m}$ wavelength range: Versatile lasers for high-power and narrow linewidth emission," in the Lasers and Electro-Optics/Quantum Electronics and Laser Science Conference (CLEO/QELS '10), May 2010.

[21] V. M. Korpijärvi, T. Leinonen, J. Puustinen, A. Härkönen, and M. D. Guina, " $11 \mathrm{~W}$ single gain-chip dilute nitride disk laser emitting around $1180 \mathrm{~nm}$," Optics Express, vol. 18, no. 25, pp. 25633-25641, 2010.

[22] Z. L. Liau, "Semiconductor wafer bonding via liquid capillarity," Applied Physics Letters, vol. 77, no. 5, pp. 651-653, 2000.

[23] J. Rautiainen, J. Lyytikäinen, A. Sirbu et al., " 2.6 W opticallypumped semiconductor disk laser operating at $1.57-\mu \mathrm{m}$ using wafer fusion," Optics Express, vol. 16, no. 26, pp. 21881-21886, 2008.

[24] J. Lyytikäinen, J. Rautiainen, L. Toikkanen et al., "1.3- $\mu \mathrm{m}$ optically-pumped semiconductor disk laser by wafer fusion," Optics Express, vol. 17, no. 11, pp. 9047-9052, 2009.

[25] A. Rantamäki, A. Sirbu, A. Mereuta, E. Kapon, and O. G. Okhotnikov, " $3 \mathrm{~W}$ of $650 \mathrm{~nm}$ red emission by frequency doubling of wafer-fused semiconductor disk laser," Optics Express, vol. 18, no. 21, pp. 21645-21650, 2010.

[26] A. Chamorovskiy, J. Rautiainen, J. Lyytikäinen et al., "Raman fiber laser pumped by a semiconductor disk laser and mode locked by a semiconductor saturable absorber mirror," Optics Letters, vol. 35, no. 20, pp. 3529-3531, 2010. 

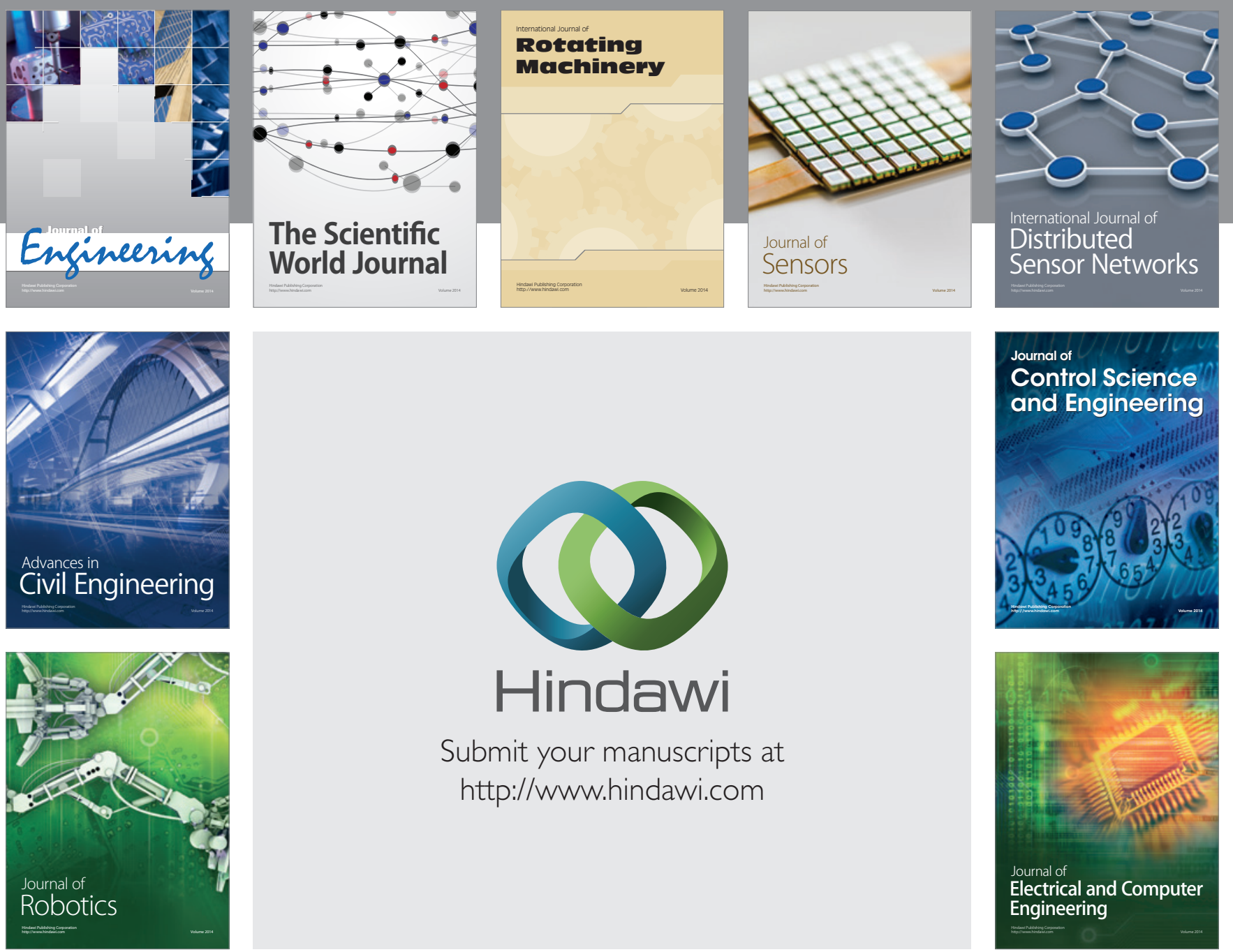

Submit your manuscripts at

http://www.hindawi.com
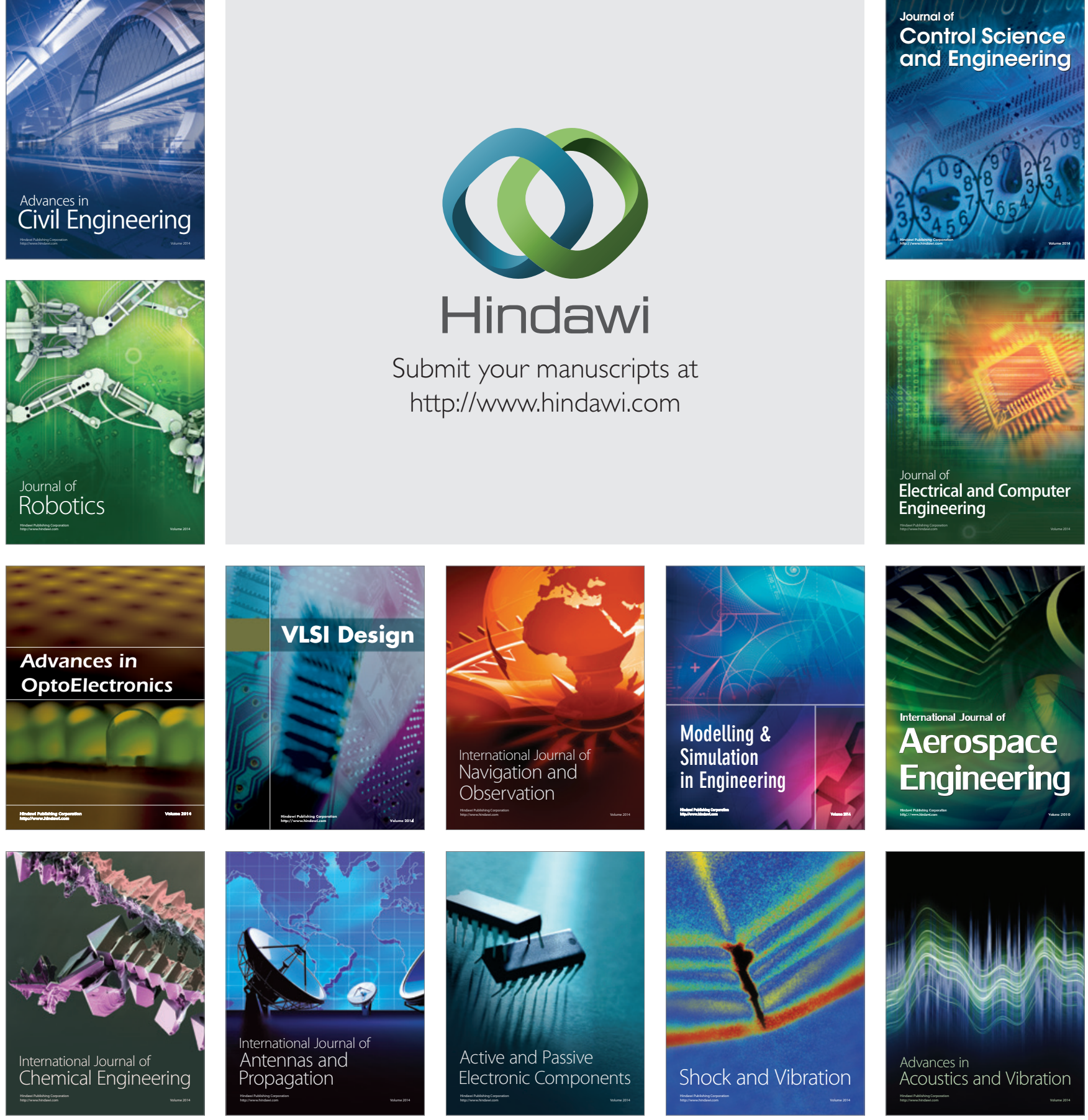